\title{
Direct measurements of the correlation between reentrant ferromagnetism and lattice expansion in $\mathrm{FeCuZr}$ alloys
}

\author{
A. Martínez, ${ }^{1}$ J. Spottorno, ${ }^{1}$ A. I. Figueroa, ${ }^{2}$ F. Bartolomé,${ }^{2}$ L. M. García, ${ }^{2}$ C. Prestipino, ${ }^{3}$ A. Hernando,,${ }^{1}$ and P. Crespo ${ }^{1}$ \\ ${ }_{1}^{1}$ Instituto de Magnetismo Aplicado, UCM-ADIF-CSIC, P.O. Box 155, 28230 Las Rozas (Madrid), Spain \\ ${ }^{2}$ Instituto de Ciencia de Materiales de Aragón and Departamento de Física de la Materia Condensada, CSIC-Universidad de Zaragoza, \\ Pedro Cerbuna 12, 50009 Zaragoza, Spain \\ ${ }^{3}$ European Synchrotron Radiation Facility, 6 rue Jules Horowitz-B.P. 220, F-38043 Grenoble, France
}

(Received 18 March 2010; revised manuscript received 16 June 2010; published 14 July 2010)

\begin{abstract}
Amorphous metastable alloy of nominal composition $\left(\mathrm{Fe}_{0.5} \mathrm{Cu}_{0.5}\right)_{87} \mathrm{Zr}_{13}$ has been synthesized by high-energy ball milling. The alloy exhibit a ferromagnetic behavior with a Curie Temperature of $T_{\mathrm{C}}=255 \mathrm{~K}$, as determined from low-field measurements whereas no transition to a paramagnetic state is observed under highenough applied magnetic fields. The evolution of hysteresis loops with temperature as well as thermoremanence measurements indicate an anomalous magnetic behavior characterized by a spontaneous increase in the magnetization values as well as by a magnetic hardening when the temperature is increased above $T_{\mathrm{C}}$. These effects are strongly correlated with a dilation of the Fe-Fe nearest-neighbor distances, as determined from extended x-ray absorption fine structure (EXAFS) studies. EXAFS results indicate an almost negligible thermal expansion at temperatures below $T_{\mathrm{C}}$ while normal thermal expansion takes place at higher temperatures. Such expansion seems to promote a reinforcement of the ferromagnetic interactions among Fe-Fe atoms that would account for the observed spontaneous increase in the magnetization as well as for the evolution of the coercive field.
\end{abstract}

DOI: 10.1103/PhysRevB.82.012406

PACS number(s): 72.20.Pa, 61.05.cj, 74.25.Ha

Invar alloys have attracted a broad interest since this effect was observed by Guillaume more than one hundred years ago in FeNi steels with around 35 at. \% Ni. ${ }^{1}$ Invar alloys are characterized by an almost negligible thermalexpansion coefficient in the ferromagnetic regime. The decrease in the magnetic moment upon increasing temperature causes a shrinking of the lattice that compensates the anharmonic thermal expansion. This behavior points out the strong interdependence of the local-magnetic configuration and atomic distances, for instance, see Ref. 2. Although Invar effect has been interpreted in terms of electronic transitions between a high-spin and high-volume state to a low-spin and low-volume state, ${ }^{3}$ recent first-principles calculations seem to identify more than two magnetic states in these alloys. ${ }^{4,5}$

Besides the thermal-expansion features, there are several physical properties of invar alloys that also exhibit an anomalous dependence with temperature, magnetic field, or pressure. Several groups have reported changes in the magnetic state induced by high pressures. Matsushita et al. ${ }^{6}$ have investigated the pressure-induced magnetic-phase transitions in some Fe-based invar alloys by means of ac susceptibility measurements. It was found that the ferromagnetic state vanished at high pressures and high-pressure magnetic states appear with pressure. Similar dependence of the Curie temperature with pressure have been obtained by means of Mössbauer spectroscopy by Abd-Elmeguid and Micklitz ${ }^{7}$ in FePt invar alloys. More recently, Nataf et al. ${ }^{8}$ have investigated $\mathrm{Fe}_{64} \mathrm{Ni}_{36}$ and $\mathrm{Fe}-\mathrm{Pt}$ invar alloys through $\mathrm{X}$-ray magnetic circular dichroism up to $12 \mathrm{GPa}$ pressure. Studies on the enhancement of the Curie temperature of invar alloys by applying tensile stress have also been reported. ${ }^{9}$

Recently, fcc-FeCu alloys have been reported to exhibit invar characteristics. ${ }^{10,11}$ By means of neutronthermodiffraction measurements an anomalous thermal de- pendence of the lattice parameter in fcc-FeCu alloys was observed. Lattice parameter in these fcc alloys are characterized by a little variation with temperature in the ferromagnetic regime whereas increasing the temperature above the Curie temperature promotes a strong increase in the thermalexpansion coefficient values. In addition, high-field susceptibility in these alloys is very large, suggesting a strong influence of magnetovolume effects in the fcc-FeCu system, i.e., large volume magnetostriction values.

In a previous work, anomalies in the thermoremanence (TRM) values were reported for amorphous $\mathrm{FeCuZr}$ and FeZr alloys. ${ }^{12,13}$ It was shown that the TRM values spontaneously increased above Curie temperature. Such enhancement in TRM values could not be due to structural changes and was tentatively attributed to thermal-expansion effects. Unfortunately, only indirect evidences arising from an increase in the intensity of neutron-diffraction peaks could be obtained.

Up to now, a direct evidence of the correlation between ferromagnetism and interatomic distances has not been reported for these invar alloys. In this work, a direct evidence of such relationship in $\left(\mathrm{Fe}_{0.5} \mathrm{Cu}_{0.5}\right)_{87} \mathrm{Zr}_{13}$ alloy is obtained by comparing two measurement carried out under a zero [extended $\mathrm{x}$-ray absorption fine-structure measurements (EXAFS)] or nearly zero, in the case of superconducting quantum interference device (SQUID) measurements, applied field. EXAFS is a technique of choice for studying nearestneighbor (NN) distances in multicomponent alloys since the energy of the radiation can be tuned for studying a particular element. ${ }^{14,15}$ In the later, it has been reported that EXAFS can provide differential interatomic-distance measurements with a resolution as high as $10^{-15} \mathrm{~m}$. This fact combined with the element selectivity of EXAFS makes this technique highly attractive for studying the thermal dependence of 
nearest-neighbor distances of a particular element.

The addition of $\mathrm{Zr}$ decreases the Curie temperature of the alloys with respect to that of fcc- $\mathrm{Fe}_{50} \mathrm{Cu}_{50}$ alloys, being $T_{\mathrm{C}}$ $=255 \mathrm{~K}$ for the $\mathrm{Zr}$ containing sample and $T_{\mathrm{C}}=550 \mathrm{~K}$ for the equiatomic composition. The fcc- $\mathrm{Fe}_{50} \mathrm{Cu}_{50}$ solid solution has been obtained by high-energy ball milling (HEBM), see, for instance, Ref. 16. In particular, $\mathrm{FeCu}(\mathrm{Zr})$ alloys exhibit an anomalous magnetic behavior for temperatures above $T_{\mathrm{C}}$ characterized by spontaneous increase in the magnetization as well as by an increase in the coercive field. ${ }^{13}$

Temperature dependence of the Fe-Fe near-neighbor distance has been studied by means of EXAFS in the $K$ edge of iron in the vicinities of $T_{\mathrm{C}}$. In this Brief Report we show by means of EXAFS spectroscopy that disordered nanocrystalline $(\mathrm{FeCu}) \mathrm{Zr}$ solid solutions constitute a family of invar alloys, presenting a behavior not reported up to present date in such systems. Disorder here means lack of long-range order even though a local fcc short-range order is detected by $\mathrm{x}$-ray diffraction. Second, EXAFS spectroscopy shows that the TRM enhancement is directly related with a dilation in Fe-Fe nearest-neighbor distances that takes place above $T_{\mathrm{C}}$, reinducing a ferromagnetic behavior above the Curie temperature of the alloys.

Metastable alloys of nominal composition $\left(\mathrm{Fe}_{0.5} \mathrm{Cu}_{0.5}\right)_{87} \mathrm{Zr}_{13}$ (at. \%) have been synthesized by HEBM. The starting materials were $\mathrm{Fe}, \mathrm{Cu}$, and $\mathrm{Zr}$ in powder form with a purity of $99.9 \%$. The final milling time was $120 \mathrm{~h}$. To avoid oxidation of the powder upon the milling process the vials were sealed under nitrogen atmosphere prior to the milling. The final composition of the powder was determined by using scanning electron microscopy equipped with an energy dispersive $\mathrm{x}$-ray analysis (EDX) whereas magnetization was determined by SQUID magnetometry.

EXAFS measurements, as a function of temperature, have been performed in BM29 beamline at the ESRF in Grenoble, France. Measurements were collected in transmission mode, at the Fe $K$ edge from 6900 to $8100 \mathrm{eV}$. After extracting the EXAFS spectra signal, they were first normalized ${ }^{17}$ to the edge-step and post-edge fitted. The EXAFS spectra were studied using the ATHENA and ARTEMIS programs from the IFEFFIT package developed by Matt Newville at consortium for Advanced Radiation Sources at the University of Chicago. ${ }^{18}$

Thermal dependence of the magnetization measured under different applied fields, ranging from 0.01 up to $5 \mathrm{~T}$, is shown in Fig. 1. The Curie temperature of the alloys has been determined from the inflection point of the thermal dependence of the magnetization measured with a low applied field of $0.01 \mathrm{~T}$. The relatively sharp decrease in the magnetization upon increasing the temperature indicates the transition from a ferromagnetic to a paramagnetic state and allows estimating the $T_{\mathrm{C}}$ values from the minimum of the derivative of the magnetization with temperature, $d M / d T$. The Curie temperature is estimated to be $255 \mathrm{~K}$, see inset Fig. 1. However, it should be noticed that such a transition is not evident when the magnetization is measured with a much higher applied field. Under $5 \mathrm{~T}$ field the experimental data follow, a $T^{3 / 2}$ law in the low-temperature regime whereas upon increasing temperature the magnetization does not fall to zero in the whole temperature range. In fact, at $300 \mathrm{~K}$ the mag-

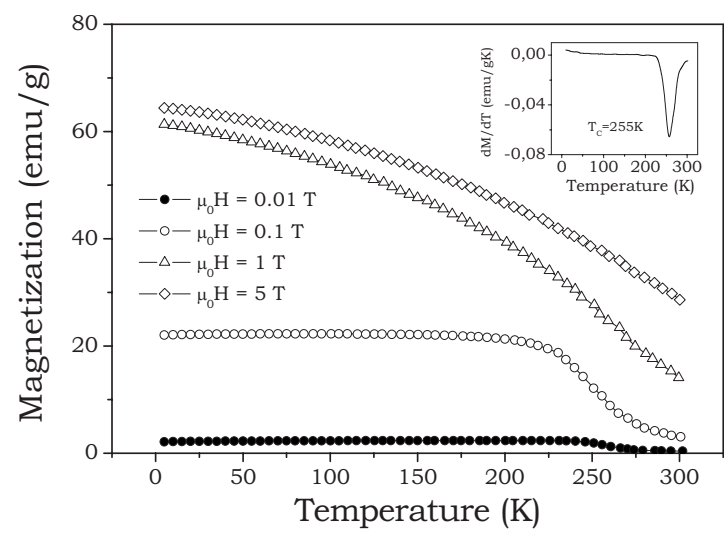

FIG. 1. Temperature dependence of the magnetization measured under different applied fields. Inset shows the derivative of the magnetization with temperature for an applied field of $0.01 \mathrm{~T}$. The Curie temperature is determined as to be $255 \mathrm{~K}$.

netization exhibits a non-negligible value. Thus, it can be concluded that the Curie temperature of the alloy exhibits a strong field dependence with applied field, increasing in at least $50 \mathrm{~K}$ when measuring at high enough applied fields. ${ }^{19}$ The second anomaly that concerns the magnetic behavior of the alloys is found in the TRM values, in particular, at the vicinity of the low-field Curie temperature. TRM is measured after cooling the sample down to $5 \mathrm{~K}$ under an applied magnetic field. Then, at $5 \mathrm{~K}$ the field is removed and the remanence is measured while the sample is heated up. Figure 2 shows the thermoremanence curves after cooling the sample under $0.01,0.1$, and $1 \mathrm{~T}$ magnetic fields.

Upon increasing temperature, TRM decreases until it reaches a minimum value at temperatures close to the $T_{\mathrm{C}}$. However, further increase in temperature promotes a spontaneous increase in the magnetization that is observed up to $300 \mathrm{~K}$. Note that such an increase takes place under a nearly and constant zero applied field and therefore it is a temperature-induced effect.

Both experimental observations, the huge-temperature dependence of the Curie temperature with applied field and the spontaneous increase in the magnetization above $T_{\mathrm{C}}$, indicate that the origin of such anomalies could rely on magnetovolume effect. In particular, the enormous shift in the $T_{\mathrm{C}}$ values is a clear indication that high enough applied field could

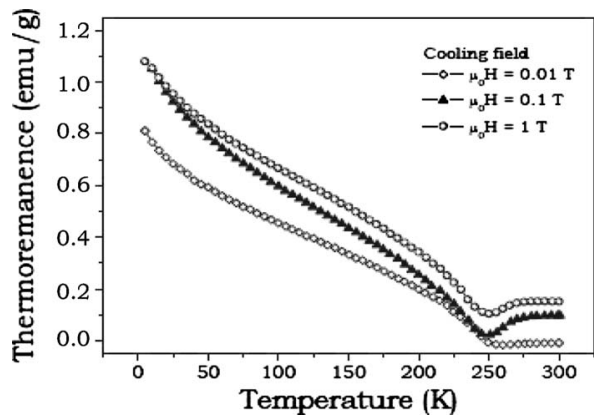

FIG. 2. TRM curves measured after cooling the sample down to $5 \mathrm{~K}$ under different magnetic fields: notice the spontaneous increase in the TRM values above the low-field Curie temperature of the sample. 

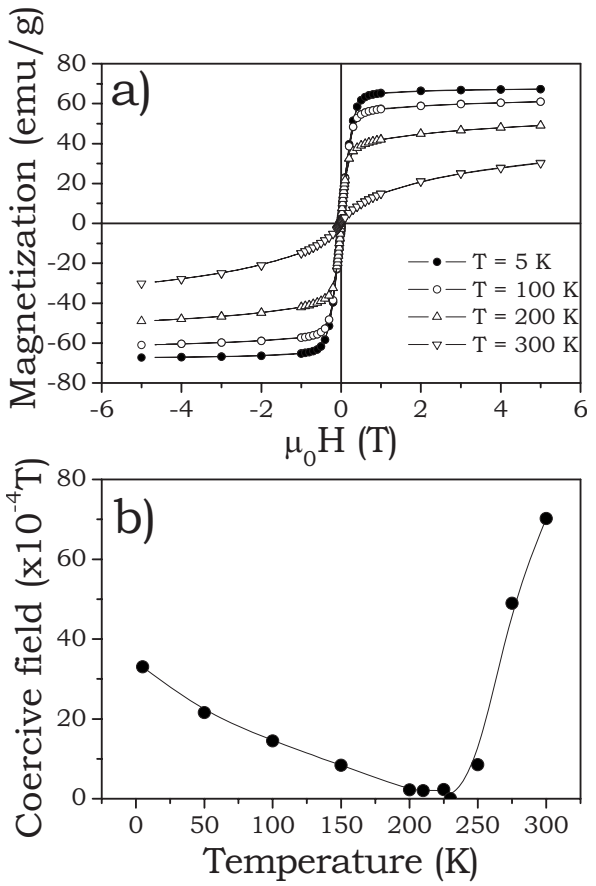

FIG. 3. (a) Hysteresis loops at some selected temperatures and (b) temperature dependence of the coercive field.

promote an expansion of the lattice, via volume magnetostriction that would reinforce $\mathrm{Fe}-\mathrm{Fe}$ ferromagnetic interactions and ferromagnetic ordering would be maintained upon, at least, room temperature (RT).

Besides both experimental results, a third anomaly appears when the thermal evolution of the coercive field $\left(H_{\mathrm{C}}\right)$ is analyzed. Hysteresis loops were measured at different temperatures from $5 \mathrm{~K}$ to RT and are shown in Fig. 3(a) whereas the evolution of $H_{\mathrm{C}}$ with temperature is plotted in Fig. 3(b). From data plotted in Fig. 3(a), it can be seen that the highfield susceptibility values are large, which again seems to indicate that the sample exhibits large volume magnetostriction values. However, the most striking behavior can be observed in Fig. 3(b). The evolution of $H_{\mathrm{C}}$ with temperature can be summarized as follows: first the $H_{\mathrm{C}}$ values decrease from $3.3 \mathrm{mT}$ upon increasing the temperature down to a nearly zero value at $225 \mathrm{~K}$. By increasing the temperature above $T_{\mathrm{C}}$, the alloy exhibits a magnetic hardening, reaching a value of around $7 \mathrm{mT}$ at $300 \mathrm{~K}$.

The experimental results show that: (i) $T_{\mathrm{C}}$ has a strong dependence with the applied field; (ii) the magnetization spontaneously increases with the temperature above $T_{\mathrm{C}}$ in absence of any applied field; and (iii) the coercive field increases with temperature for $T>T_{\mathrm{C}}$, where $T_{\mathrm{C}}$ is the Curie temperature determined from low-field magnetization measurements, i.e., the alloy exhibits a ferromagnetic behavior characterized by magnetic hysteresis is observed above $T_{\mathrm{C}}$. All results point out that above $T_{\mathrm{C}}$ a reinforcement of the ferromagnetic interactions among $\mathrm{Fe}$ atoms is taking place, i.e., the ferromagnetic behavior is recovered.

EXAFS experiments have been performed at the $\mathrm{Fe} K$ edge for obtaining the evolution of the Fe-Fe nearestneighbor distance as a function of temperature. The data obtained indicate two different regimes with temperature, see

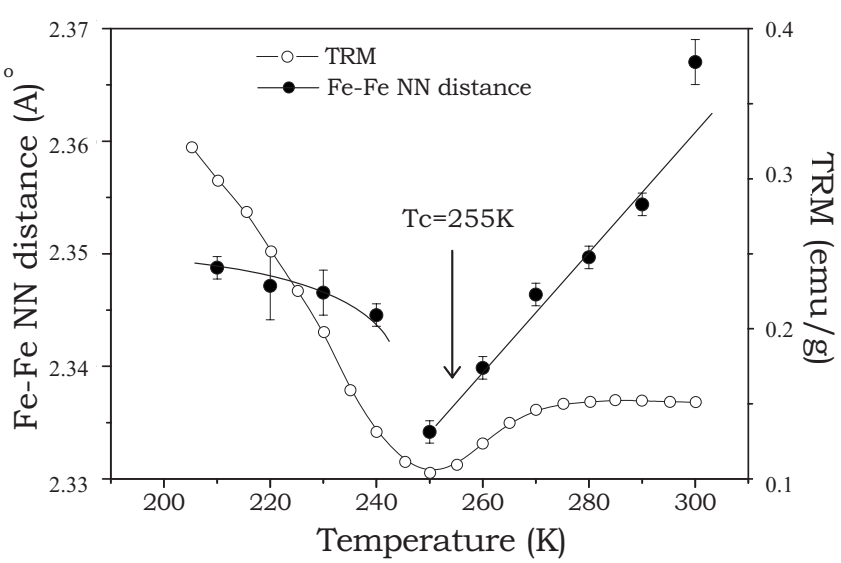

FIG. 4. Temperature dependence of the Fe-Fe nearest-neighbor distances (solid lines) compared with the thermoremanence evolution after cooling the sample under $1 \mathrm{~T}$ applied field (open symbols).

Fig. 4: (i) at temperatures well below $T_{\mathrm{C}}$, the Fe-Fe NN distances are almost independent of temperature, thus indicating that alloy exhibits invar behavior; (ii) when the temperature is approaching $T_{\mathrm{C}}$ a decrease in the Fe-Fe NN distances is clearly observed; and (iii) for temperatures above $T_{\mathrm{C}}$ a strong change in the thermal dependence of Fe-Fe NN is observed. From these data the linear-expansion coefficient can be estimated as to be $4 \times 10^{-5} \mathrm{~K}^{-1}$ in the latter regime. Moreover, the comparison of the evolution of $\mathrm{Fe}-\mathrm{Fe} \mathrm{NN}$ with that of the TRM values clearly shows that there is a strong correlation between the magnetic moment and Fe-Fe NN distances. In invar systems, the temperature dependence of the volume magnetostriction compensates the thermal expansion. The apparent zero or negative thermal expansion in the ferromagnetic regime observed below $T_{\mathrm{C}}$ in Fig. 4 is caused by the opposite effects of anharmonic thermal expansion and the contraction associated with the decrease in the spontaneous magnetization. When $T$ is approaching $T_{\mathrm{C}}$, the Fe-Fe NN drop since the strong decrease in the magnetization cannot be compensated by thermal expansion at this temperature range. Once the ferromagnetic to paramagnetic transition has taken place, the thermal expansion dominates and the Fe-Fe NN distances exhibit a rapid increase with temperature.

The increase in Fe-Fe NN distances is directly correlated with the spontaneous increase in the TRM values. Thus, by increasing the $\mathrm{Fe}-\mathrm{Fe} \mathrm{NN}$ distances, the most stable magnetic configuration corresponds to a ferromagnetic interaction between $\mathrm{Fe}$ atoms. Once the invar effect is not effective, the thermal expansion allows recovering $\mathrm{Fe}-\mathrm{Fe} \mathrm{NN}$ distances at which the ferromagnetic configuration is the lower energy configuration and room-temperature ferromagnetism, characterized by magnetic hysteresis with non-negligible coercive field is recovered. These results are in clear agreement with the dependence on the $\mathrm{Fe}-\mathrm{Fe} \mathrm{NN}$ distances of the magnetic properties pointed out by Wasserman on invar alloys. By comparing the Curie temperature and magnetic moment with the lattice parameter in different compounds it was shown that the ferromagnetic interaction among Fe-Fe becomes unstable as the atomic volume decreases, being $0.255 \mathrm{~nm}$ a kind of critical distance for Fe-Fe NN. For instance, it has 
been reported that the disappearance of ferromagnetism in amorphous $\mathrm{La}\left(\mathrm{Fe}_{0.85} \mathrm{Al}_{0.15}\right)_{13}$, where $\mathrm{Fe}-\mathrm{Fe} \mathrm{NN}$ distances are very close to $0.255 \mathrm{~nm}$ under high-pressure conditions. ${ }^{20} \mathrm{In}$ the case of disordered $\mathrm{FeCu}(\mathrm{Zr})$ alloys, an enhancement of the ferromagnetic interactions is clearly observed for $\mathrm{Fe}-\mathrm{Fe}$ NN distances on the order of $0.234 \mathrm{~nm}$, a value slightly smaller than that reported in Ref. 2. This difference in the $\mathrm{Fe}-\mathrm{Fe}$ values could be related with the disordered nature of the $\mathrm{FeCu}(\mathrm{Zr})$ nanocrystalline solid solution. By increasing the temperatures above the low-field $T_{\mathrm{C}}$, i.e., when the lattice expansion is not inhibited by the temperature dependence of the volume magnetostriction, the increase in Fe-Fe NN distances promotes the spontaneous increase in the TRM values.

It is important to notice that the results presented in this work show a direct evidence of the interrelationship that exists between magnetic ordering and local arrangement of magnetic atoms, in particular, nearest-neighbor distances between magnetic atoms. The increase in $\mathrm{Fe}-\mathrm{Fe}$ interatomic distances promote an increase in the density of states that reinforces ferromagnetism. ${ }^{21,22}$

In $\mathrm{FeCuZr}$ invar alloy, the normal thermal dilation occurs when the temperature is increased above the low-field Curie temperature whereas it occurs whenever a high enough applied magnetic field is applied to the alloy. Notice that in the temperature-dependent magnetization curves, the magnetization never reaches a zero value, even for the lowest applied field values.

In summary, $\left(\mathrm{Fe}_{0.5} \mathrm{Cu}_{0.5}\right)_{87} \mathrm{Zr}_{13}$ alloys obtained by HEBM, exhibit an anomalous magnetic behavior as evidenced by the strong dependence of the magnetic-order magnetic-disorder transition, the increase in thermoremanence as well as coercive field values above the low-field Curie temperature. EXAFS measurements indicate that iron interatomic distances remain almost invariant with temperature for $T<T_{\mathrm{C}}$ while above $T_{\mathrm{C}}$ the normal thermal expansion takes place. The normal expansion is directly related with the increase in TRM and $H_{\mathrm{C}}$ values. A direct experimental evidence of the connection between magnetic ordering and interatomic magnetic moments distances is shown. The dilation of the Fe-Fe NN distances that occurs above low-field $T_{\mathrm{C}}$ promotes a recovery of the ferromagnetism in these invar alloys.

This work was supported by Spanish MICINN under Projects No. MAT2009-14741-C02-01 and No. MAT200801077, and Consolider-Ingenio in Molecular Nanoscience under Project No. CSD2007-00010; and DGA by means of IMANA and CAMRADS. A.I.F acknowledges a CSIC-JAE Predoc grant.
${ }^{1}$ C. E. Guillaume, C. R. Acad. Sci. Paris 125, 235 (1897).

${ }^{2}$ E. F. Wassermann, J. Magn. Magn. Mater. 100, 346 (1991).

${ }^{3}$ R. J. Weiss, Proc. Phys. Soc. London 82, 281 (1963).

${ }^{4}$ M. van Schilfgaarde, I. A. Abrikosov, and B. Johansson, Nature (London) 400, 46 (1999).

${ }^{5}$ I. A. Abrikosov, A. E. Kissavos, F. Liot, B. Alling, S. I. Simak, O. Peil, and A. V. Ruban, Phys. Rev. B 76, 014434 (2007), and references therein.

${ }^{6}$ M. Matsushita, Y. Miyoshi, S. Endo, and F. Ono, Phys. Rev. B 72, 214404 (2005); M. Matsushita, S. Endo, K. Miura, and F. Ono, J. Magn. Magn. Mater. 269, 393 (2004).

${ }^{7}$ M. M. Abd-Elmeguid and H. Micklitz, Phys. Rev. B 40, 7395 (1989).

${ }^{8}$ L. Nataf, F. Decremps, J. C. Chervin, O. Mathon, S. Pascarelli, J. Kamarád, F. Baudelet, A. Congeduti, and J. P. Itié, Phys. Rev. B 80, 134404 (2009).

${ }^{9}$ P. Gorria, D. Martínez-Blanco, M. J. Pérez, J. A. Blanco, A. Hernando, M. A. Laguna-Marco, D. Haskel, N. Souza-Neto, R. I. Smith, W. G. Marshall, G. Garbarino, M. Mezouar, A. Fernández-Martínez, J. Chaboy, L. Fernandez Barquín, J. A. Rodriguez Castrillón, M. Moldovan, J. I. García Alonso, J. Zhang, A. Llobet, and J. S. Jiang, Phys. Rev. B 80, 064421 (2009).

${ }^{10}$ P. Gorria, D. Martínez-Blanco, and J. A. Blanco, Phys. Rev. B. 69, 214421 (2004).

${ }^{11}$ M. Eisenbach and G. M. Stocks, J. Appl. Phys. 105, 07 E509 (2009).

${ }^{12}$ A. Hernando P. Crespo, F. J. Castaño, J, Arcas, M. Multigner, J. M. Barandiarán, and L. Fernández-Barquín, Phys. Rev. B 61, 3219 (2000).

${ }^{13}$ P. Crespo M. Multigner, F. J. Castaño, R. Casero, A. Hernando,
A. García Escorial, L. Schultz, and S. N. Kaul, Phys. Rev. B 61, 14346 (2000).

${ }^{14}$ V. G. Harris, K. M. Kemmer, B. N. Das, N. C. Koon, A. E. Ehrlich, J. P. Kirkland, J. C. Woicik, P. Crespo, A. Hernando, and A. Garcia-Escorial, Phys. Rev. B 54, 6929 (1996).

${ }^{15}$ R. F. Pettifer, O. Mathon, S. Pascarelli, M. D. Cooke, and M. R. J. Gibbs, Nature (London) 435, 78 (2005).

${ }^{16}$ P. Crespo, A. Hernando, R. Yavari, O. Drbohlav, A. García Escorial, J. M. Barandiarán, and I. Oru'e Phys. Rev. B 48, 7134 (1993).

${ }^{17}$ W. T. Elam, J. P. Kirkland, R. A. Neiser, and P. D. Wolf, Phys. Rev. B 38, 26 (1988).

${ }^{18} \mathrm{http} / / / \mathrm{cars} 9$. uchicago.edu/ifeffit/

${ }^{19}$ Possible magnetic properties fluctuation associated with structural disorder are generally averaged by exchange interactions, see, for instance, J. Arcas, A. Hernando, J. M. Barandiarán, C. Prados, M. Vázquez, and P. Marín Phys. Rev. B 58, 5193 (1998); A. Hernando, I. Navarro, and J. M. González, Europhys. Lett. 20, 175 (1992).

${ }^{20}$ M. Matsushita, M. Ohta, A. Fujita, K. Fukamichi, H. Ogiyama, and F. Ono, J. Alloys Compd. 455, 21 (2008).

${ }^{21}$ H. Chen, N. E. Brener, and J. Callaway, Phys. Rev. B 40, 1443 (1989); V. L. Moruzzi and P. M. Marcus, ibid. 39, 471 (1989).

${ }^{22}$ A. Hernando, E. Navarro, M. Multigner, A. R. Yavari, D. Fiorani, M. Rosenberg, G. Filoti, and R. Caciuffo, Phys. Rev. B 58, 5181 (1998); B. Sampedro, P. Crespo, A. Hernando, R. Litrán, J. C. Sánchez-López, C. López-Cartes, A. Fernández, J. Ramírez, J. González-Calbet, and M. Vallet, Phys. Rev. Lett. 91, 237203 (2003). 